the fact that the spirochata pallida is not found in the earlier foetuses, and yet syphilitic women often have alternating abortions and still-births. Dr. F. W. Mott showed this conclusively in his interesting epidiascope demonstration at the discussion at this section in April, 1914, following an address by myself on " The Need for Research in Antenatal Pathology," and those who doubt that syphilis causes abortion should read his remarks in our Proceedings for that month. ${ }^{\bar{s}}$ If in these cases still-births are proved to be syphilitic, surely it is logical to assume that the abortions in the same women are due to the same cause, even though search for the spirochæta is negative. Those who hold these new views admit that toxic albuminuria in pregnancy may cause foetal death during both early and later pregnancy, and that in the later months the fotus may become macerated. The evidence in the case of the mother, known to have syphilis by a positive Wassermann reaction, does not seem to me to be different from that in the case of the woman who is known to be suffering from a toxic albuminuria. In both cases the mother has a disease which may destroy foetal life at all stages. I cannot help thinking that the existence of spores in the life-history of the Spirocheta pallida, as described by McDonagh, may be an explanation of the failure to find spirochrtes in abortions, the spirochrtes being destroyed by the ferments of the chorionic villi, whilst the spores escape.

Whether, therefore, the question of medical supervision during pregnancy be considered from the points of view of the welfare of the mother and unborn child, or as an educational stimulus to the nation, or from the standpoint of the increase of pathological, chemical, and therapeutical knowledge for the pro'ession, there can surely be no real difference of opinion that every pregnant woman should be seen by a doctor, and then have such supervision as her condition requires.

Manchester-square $\mathbf{W}$.

\section{TWO FATAL CASES OF METASTATIC GAS GANGRENE.}

BY KENNETH TAYLOR, M.A., M.D.,

DIRECTOR OF IABORATORIES, ROBERT WALTON GOELET RESEARCH FUND, HÔPITAI COMPLFMENTAIRE V.R. 76, FRANCK.

THe following two cases are reported because of their singularity and because of the light which ther may throw upon one of the methods of extension of infection by the B. aerogenes eapsulatus.

CASE 1.-A private, wounded 10 days previously by shell fragment. First dressing applied within a few hours. Admitted to the hospital with diagnosis of a perforating wound of right arm with fracture of the bumerus at its lower third and gaseous gangrene of the arm. Examination of the arm showed marked swelling and tension of the muscle fascia; purplish discolouration of the skin reaching from the snoulder to the lower third of forearm; several blebs over triceps muscle; diffuse subcutaneous emphysema extending from the shoulder well on to the forearm. Radial pulse could not he felt. Operation : open-flap amputation just below the shoulder-joint was performed immediately. Tissues at site of amputation showed gas in subcutaneous tissue and muscle. The muscles about the shoulderjoint were also incised and found to be gaseous, pale brickred in colour, and dry. As far as possible the gangrenous portions of these muscles were excised. Patient was placed in a sitting position. His general condition improved steadily for five days, when, although the infection about the shoulder-joint appeared to be arrested, he again showed signs of acute intoxication, and died on the sixth day after operation. Blood taken from the median basilic vein of left arm three days before death was sterile. Necropsy performed five hours after death showed no evidence of further extension of the infection on to the trunk. Examination of the limbs showed gaseous gangrene of the right gluteus maximus and medius, where sustained pressure had resulted from the sitting posture. The infection had extended to the adductor muscles of the right thigh. Cultures of heart's blood, muscles of the shoulder and of right gluteal muscles showed pure culture of the B. aerogenes capsulatus.

CASE 2.-A private, wounded six days previously by shell fragment. Was dressed one hour after injury, transported by motor to field ambulance, where a piece of shell was removed from the right gluteal muscles. He was evacuated

5 Proe. Obst. Sect., Roy. Soc. Med., 1914, p. 288. the next day by train. When admitted to the hospital patient was lying on his left side and stated that he had been forced to maintain this posture because of the position of the wound in the right buttock. On the right buttock was a large excavating wound which had evidently been incised. Cultures from this wound showed the $B$. aerogenes capsulatus. The following day gaseous distension of the right gluteus maximus was evident. An operation was performed, the gluteal muscles being widely opened. The following day, however, his general condition was worse, and further examination showed gaseous distension of the left thigh-upon which he had been resting continuously. There was no subcutaneous crepitation, and no blebs or discolouration of the skin, but tension of the fascia sheaths was evident and the thigh was tympanitic to percussion. An operation was performed: the right gluteal muscles were again examined and the exposed necrotic portions excised. The infection appeared to be limited to the region of the wound. A longitudinal incision was made through the fascia in the outer surface of the left thigh from the level of the great trochanter to $5 \mathrm{~cm}$. above the knee-joint. Through this the vastus externus muscle immediately bulged; it was incised but appeared contractile and normal. A similar incision was made on the inner surface of the left thigh, when the muscles exposed bulged out through the wound. The muscles seemed to be in healthy condition. The patient, however, became more and more toxic, and died 24 hours later-eight days after injury and two days after admission to the hospital. Necropsy showed no extension of the gangrenous process in the right gluteal muscles, the infection being limited to the tissues exposed in the wound. There was no communica tion between the wound of the right gluteal muscles and the tissues involved in the left thigh or left gluteal region. Careful examination of the subcutaneous tissue and muscles intervening showed no evidence whatever of infection. Subcutaneous crepitation was noted over the left thign and les nearly to the ankle-joint and upwards over the anterior surface of the left half of the abdomen well above the crest of the ilium. The left half of the scrotum was distended with gas. There was no discolouration of the skin and no blebs. Examination of the left thigh showed extensive necrosis of large muscle masses, chiefly limited to the three glutei, the rotator muscles of the thigh, and the adductors. The other muscles appeared to be intact. Cultures from the operative wounds of left thigh and gaseous subcutaneous tissue above the crest of the left ilium showed the Bacillus aerogenes capsulatus. Cultures of the gaseous subcutaneous tissue over the gastrocnemius muscle on the left leg remained sterile. Culture of the heart's blood was sterile.

These two cases are similar in the following respects: In both the focus of the infection and the gangrene which was the final cause of death were of metastatic origin-e.g. there was certainly no direct extension from the wound to the area of muscle destruction. In the first, blood culture three days before death proved negative; in the second, culture from the heart's blood at necropsy was likewise negative. ${ }^{1}$ In both the terminal gangrene occurred over a week after injury. In both the site of the metastatic gangrene was that upon which continuons pressure ${ }^{2}$ was exerted by the posture which the patient was obliged to maintain. Apparently direct extension through subcutaneous tissue, muscles, and muscle fascia could be excluded. Extension by superficial or deep lymphatics seems to have been unlikely. The results of the blood cultures gave no proof of any true, continuous bacteriæmia, but the blood stream appeared to be the most probable mode of extension.

The conclusions to be drawn from the two cases appear, therefore, to be :-

1. That blood invasion may occur during the life of the patient but is probably only of short duration.

2. That the vitality of a muscle mass may be so lowered by continuous subjection to pressure and the resultant interference with its circulation that it may become a site for the fixation and activity of the bacilli following the temporary invasion of the blood.

3. That it is advisable, in the case of a patient who has a wound infected by the gas bacillus, to make a careful examination of the entire body and not limit the attention to the region of the wound.

4. That it is dangerous to allow a patient suffering from a wound infected by the gas bacillus to maintain a fixed position in bed.

1 This is not unusual in necrops es performed soon af'er death. Other cases are reported in the following paper: "O nservations on the Patnology and Bacteriology of Gas Gangrene." Journal of Patholngy and Bacteriology, 1916, xx., 384 . 2."Factors Responsible for Gaseous Gangrene," Tre Lakcet,
Jan. 15th, 1916. 